\title{
DISTRIBUIÇÃO ESPACIAL DE CARBONO EM SOLO SOB FLORESTA PRIMÁRIA NA AMAZÔNIA MERIDIONAL'
}

João Paulo Novaes Filho², Evandro Carlos Selva², Eduardo Guimarães Couto², Johannes Lehmann², Mark S. Johnson ${ }^{3}$ e Susan J. Riha ${ }^{3}$

\begin{abstract}
RESUMO - O estudo do carbono assume grande importância devido à sua estreita relação com as mudanças climáticas da Terra. Uma das causas relevantes dessas alterações é a rápida substituição das florestas tropicais da Amazônia por sistemas agropecuários. Este trabalho foi desenvolvido em Juruena (MT), com os seguintes objetivos: estudo da distribuição espacial do teor de carbono e a estimativa do seu estoque na camada de 0$0,60 \mathrm{~m}$ de solo em microbacias sob floresta primária, por meio de técnicas geoestatísticas. Foram demarcados 185 pontos georreferenciados em forma de malha sistemática regular, abrangendo quatro microbacias próximas, com espaçamento de $20 \times 20 \mathrm{~m}$, em que foram coletadas amostras de solo até a profundidade de $0,60 \mathrm{~m}$. A densidade aparente aumentou de $1,36 \pm 0,081 \mathrm{~g} \cdot \mathrm{cm}^{-3}$ ( \pm desvio-padrão) para 1,46 $\pm 0,083 \mathrm{~g} . \mathrm{cm}^{-3}$, nas profundidades de 0-0,20 m e 0,40-0,60 m, respectivamente. As microbacias apresentaram estoques médios de carbono (profundidade de 0-0,60 m) distribuídos da seguinte forma: microbacia $1=56,73 \mathrm{t}_{\text {ha }}{ }^{-1}$, microbacia $2=59,35 \mathrm{t} \mathrm{ha}^{-1}$, microbacia $3=59,22$ t.ha $^{-1}$ e microbacia $4=64,35$ t.ha ${ }^{-1}$, com média geral de 59,74 $\pm 10,30$ t.ha $^{-1}$. Apesar de existir uma aparente homogeneidade quanto às características visíveis na paisagem, como relevo, cor de solo e vegetação, os atributos carbono e argila apresentaram considerável variabilidade, mesmo dentro de pequenos espaços como as microbacias estudadas. Isso demonstrou que a extrapolação de dados para estimativas de estoque de carbono em áreas maiores pode projetar resultados falsos, quando a variabilidade espacial de atributos de solo não é levada em consideração.
\end{abstract}

Palavras-chave: Geoestatística, krigagem e dependência espacial.

\section{SPATIAL DISTRIBUTION OF SOIL CARBON UNDER PRIMARY FOREST COVER IN THE SOUTHERN AMAZON}

\begin{abstract}
The study of the carbon cycle is of great importance due to its intricate relationship with changes in the earth's climate. One of the relevant causes of changes in the carbon cycle is the high rate of land use change in the Amazon region, which replaces tropical forest cover with agriculture and grazing. This study was carried out in Juruena, Mato Grosso with objective of studying the spatial distribution of soil carbon concentrations and stocks $(0-0.60 \mathrm{~m})$ in headwater catchments under primary forest using geostatistical techniques. A total of 185 georeferenced sample points were established using a $20 \times 20 \mathrm{~m}$ grid over four headwater catchments. Soil samples were collected from the 0-0.60 m depths at each point. The bulk density increased from $1.36 \pm 0.08 \mathrm{~g} \mathrm{~cm}^{-3}$ (mean $\pm 1 \mathrm{SD}$ ) to $1.46 \pm 0.083 \mathrm{~g} \mathrm{~cm}^{-3}$ for the $0-0.20$ and $0.40-0.60 \mathrm{~m}$ depths, respectively.
\end{abstract}

\footnotetext{
${ }^{1}$ Recebido em 01.06.2006 e aceito para publicação em 20.10.2006.

${ }^{2}$ Departamento de Solos e Extensão Rural da UFMT, Av. Fernando Corrêa da Costa, s/n, CEP 78080-0900, Cuiabá-MT. E-mail:<jpnovaes@terra.com.br>,<evandroc@cpd.ufmt.br>e<couto@cpd.ufmt.br>.

${ }_{3}^{3}$ Department of Crop and Soil Sciences, Cornell University, Ithaca, NY 14850, USA. E-mail: <c1273@ cornell.edu>, <msj8@cornell.edu>e<sjr4@cornell.edu>.
} 
Soil carbon stocks $(0-0,60 \mathrm{~m})$ were distributed between the headwater catchments as: basin $1=56.73$ $t h a^{-1}$, basin $2=59.35 t h a^{-1}$, basin $3=59.22 t h a^{-1}$ and basin $4=64.35 t h a^{-1}$; with a mean for the entire study area of $59.74 \pm 10.30 \mathrm{tha}^{-1}$. In spite of the apparent homogeneity of the visible landscape characteristics such as slope, soil color and vegetation, the carbon and soil clay attributes were found to vary greatly. This variability over small distances demonstrates that extrapolation of soil characteristics and soil carbon stocks to larger areas could produce erroneous results if the spatial variability of the soil attributes is not taken into consideration.

Keywords: Geostatistics, kriging and space dependence.

\section{INTRODUÇÃO}

A Amazônia, com sua grande diversidade geológica, aliada ao relevo e clima, é constituída por várias classes de solos (EMBRAPA, 1999; LAURANCE et al., 1999), o que resulta em diferentes potenciais de armazenagem de carbono. Entender e quantificar o conteúdo nos diferentes compartimentos solo, planta e atmosfera ajudam as estimativas mais confiáveis do balanço regional e global de carbono.

De acordo com Batjes e Dijkshoorn (1999), as principais classes de solos da Amazônia são os Argissolos e Latossolos, que representam 24,8 e 24,6\% da área total, com média do estoque de carbono do solo (profundidade de 0-0,30 m) de 44,00 e 50,50 t.ha-1, respectivamente.

No sudoeste da Bacia Amazônica, Moraes et al. (1996), estudando atributos de solo, observaram que, apesar das variações nos teores de argila, o estoque de carbono do solo sob vegetação de floresta é de 37,00 t.ha-1 ${ }^{-1}$ considerando-se uma profundidade de 0-0,30 m; e a densidade aparente variou de 1,30 g.cm ${ }^{-3}$ nos primeiros $0,05 \mathrm{~m}$ até $1,51 \mathrm{~g} . \mathrm{cm}^{-3}$ na profundidade de $0,20-0,30 \mathrm{~m}$.

A estimativa do estoque de carbono na região amazônica tem sido feita em diferentes locais, com resultados também muito variados. Melo (2003), por exemplo, estimou o estoque de carbono nos solos do Estado do Acre, encontrando variações de 30,00 a 36,00 t.ha ${ }^{-1}$ em Argissolos e 33,00 a 42,00 t.ha ${ }^{-1}$ em Latossolos, na profundidade de $0-0,30 \mathrm{~m}$; e densidade aparente entre 1,10 g. $\mathrm{cm}^{-3}$ nos horizontes superficiais e 1,50 g. $\mathrm{cm}^{-3} \mathrm{em}$ profundidade. Variações semelhantes foram verificadas por Neill et al. (1997) em alguns municípios de Rondônia, onde o estoque de carbono na camada de $0-0,30 \mathrm{~m}$ do solo variou de 32,30 $\pm 3,20$ t.ha ${ }^{-1}$ (Nova Vida) a 62,00 $\pm 2,30$ t.ha ${ }^{-1}$ (Porto Velho).

R. Árvore, Viçosa-MG, v.31, n.1, p.83-92, 2007
Também no Estado de Rondônia, Cerri et al. (2004b) estimaram estoques de carbono de solo sob floresta, utilizando modelagem geoestatística, e observaram variações de 24,00 a 26,00 t.ha ${ }^{-1}$, na profundidade de 0-0,20 m. Na região noroeste do Estado de Mato Grosso, o mapa elaborado por Bernoux et al. (2002), usando dados do Radambrasil e IBGE, registram 45,00 t.ha ${ }^{-1}$ de carbono na camada de 0-0,30 m. Entretanto, esses mapas de escalas pequenas ainda não representam a real variação dos dados das florestas da porção sul da Amazônia.

Para a o estudo de uso do solo e comparação de paisagens de ecossistemas, Stein e Ettema (2003) evidenciaramm a exigência da aplicação de estatísticas não clássicas, principalmente no caso de interpolação de variáveis espacialmente dependentes. Segundo o modelo estatístico clássico, as variações são aleatórias, além de não considerar a correlação espacial relativa aos locais das amostras (TRANGMAR et al., 1985). Nesse contexto, os objetivos deste trabalho foram estudar a distribuição espacial do teor de carbono e estimar o seu estoque na camada de 0-0,60 m de solo sob floresta primária, por meio de técnicas geoestatísticas.

\section{MATERIAL E MÉTODOS}

\subsection{Caracterização da Área}

A pesquisa foi conduzida na Fazenda Rohsamar, localizada no Município de Juruena, região noroeste do Estado de Mato Grosso (latitude 10²8’35" S e longitude $58^{\circ} 28^{\prime} 10^{\prime \prime}$ WGr.). Essa região integra a parte meridional da grande Bacia Amazônica, sendo a área experimental constituída por quatro microbacias sob vegetação de floresta não perturbada, com dimensões respectivas de: microbacia $1=1,12$ ha; microbacia $2=1,90$ ha; microbacia $3=1,09$ ha; e microbacia $4=1,05$ ha. A sua vegetação é classificada como de Floresta Ombrófila Densa de formação submontana, com um grande número de palmeiras próximo aos córregos (IBGE, 1990). 
Segundo a classificação de Köppen, o tipo climático predominante na região é o Awi - clima tropical úmido, com estação seca bem definida (inverno/verão), com temperatura média anual de $24{ }^{\circ} \mathrm{C}$ e precipitação pluviométrica média de $2.200 \mathrm{~mm}$ anual. A geologia é composta pela unidade denominada Complexo Xingu, e a formação geomorfológica pertence à Depressão Interplanáltica da Amazônia Meridional, com relevo suave-ondulado e altitude em torno de $260 \mathrm{~m}$ (BRASIL, 1980).

Os solos das microbacias são classificados como Argissolo Vermelho Distrófico plíntico, com inclusão de Latossolo Vermelho-Amarelo Distrófico típico e Argissolo Vermelho-Amarelo Distrófico plíntico, definidos em levantamento pedológico realizado por Couto e Oliveira (2003), na escala de 1:100.000. Nas microbacias 1 e 2 foram encontradas as três classes pedológicas citadas anteriormente, e nas microbacias 3 e 4 observou-se a predominância de Latossolo VermelhoAmarelo Distrófico típico.

\subsection{Sistema de Amostragem}

As quatro microbacias foram selecionadas pela proximidade entre si, formando uma seqüência de 1 a 4 e demarcadas com estacas, formando um conjunto de 185 pontos arranjados em malha regular de 20 × 20 $\mathrm{m}$. Esses locais demarcados foram georreferenciados (coordenadas planas UTM) e as cotas, determinadas por meio de levantamento altimétrico com nível óptico (referencial de altitude tomado com altímetro barométrico). O número de pontos amostrais nas bacias 1, 2, 3 e 4 ficou distribuído, respectivamente, da seguinte forma: 43 (38,4 amostras por ha), 65 (34,2 amostras por ha), 39 (35,8 amostras por ha) e 38 (36,2 amostras por ha).

A coleta de amostras foi realizada com a utilização de trado-holandês em cada ponto da malha até a profundidade de $0-0,60 \mathrm{~m}$. Na primeira profundidade (0-0,20 m), foram coletadas amostras compostas, sendo cada uma obtida pela mistura de 5 subamostras, dentro do raio de $1 \mathrm{~m}$ do ponto selecionado; nas outras profundidades, a coleta foi de amostras simples. Para a determinação da densidade aparente, foram retiradas amostras de solo indeformadas com amostrador de Kopecky, também até a profundidade de 0-0,60 m.

\subsection{Atributos Estudados e Métodos de Análises}

As amostras foram secadas ao ar e passadas por peneira de malha de $2 \mathrm{~mm}$ de abertura, e a análise de carbono foi feita pelo método de combustão por via seca, sob alta temperatura (forno Eltra, $1.350^{\circ} \mathrm{C}$ ), em analisador Multi NC 3.000 (Analytik Jena, Germany).

A análise textural foi determinada pelo método da pipeta com agitação lenta por 16 horas (30 rpm), dispersa com $\mathrm{NaOH}$ e hexametafosfato de sódio (CAMARGO et al., 1986). A densidade aparente foi determinada por amostras não deformadas, retiradas por anéis de aço com volume interno de $50 \mathrm{~cm}^{3} \mathrm{e}$ secadas na estufa a $105^{\circ} \mathrm{C}$, por 24 e 48 horas (EMBRAPA, 1997).

Os estoques de carbono foram obtidos pela multiplicação da concentração do elemento pela densidade aparente da camada de solo para expressar a relação peso/volume. Os valores foram estimados no perfil de $0-0,60 \mathrm{~m}$ de profundidade e apresentados na unidade t.ha-1.

\subsection{Métodos Estatísticos}

As análises estatísticas foram realizadas em duas etapas. A primeira consistiu da organização dos dados, aplicando-se a estatística descritiva e o teste de média (Tukey, $\alpha=0,05$ ), realizados por meio do programa SPSS 10 (SPSS, 1999). Ainda sob a ótica da estatística convencional, a classificação da variabilidade dos atributos de solo foi realizada pelo estudo do coeficiente de variação (WILDING e DREES, 1983). O cálculo do número de amostras $(n)$ necessário, segundo Davis et al. (1995), para estimar a média da população dentro do limite de precisão estipulado foi obtido pela equação:

$$
n=t^{2} C V^{2} / p^{2}
$$

em que $C V$ é o coeficiente de variação, $t=1,96$ (empregando $95 \%$ do intervalo de confiança, quando $n=\infty)$, e $p=$ porcentagem de erro provável $(P=0,10$, implica a aceitação de $\pm 10 \%$ de erro). Na segunda etapa, foram aplicadas funções geoestatísticas (semivariograma e krigagem ordinária) para analisar a dependência espacial do carbono no solo e produzir mapas de interpolação, utilizando-se os programas Gamma Design (ROBERTSON, 2000) e Surfer 8.0 (SURFER, 2002). O semivariograma foi determinado pelo algoritmo:

$$
\gamma^{*}(h)=\frac{1}{2 N(h)} \sum_{i=1}^{N(h)}\left[Z\left(x_{i}\right)-Z\left(x_{i}+h\right)\right]^{2}
$$

em que $\gamma^{*}(h)$ é o semivariograma estimado, $N(h)$ é

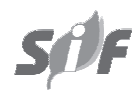

R. Árvore, Viçosa-MG, v.31, n.1, p.83-92, 2007 
o número de pares de valores medidos $Z\left(x_{i}\right), Z\left(x_{i}+h\right)$, separados por um vetor distância $h$. O interpolador de krigagem ordinária foi dado por:

$$
Z^{*}\left(x_{0}\right)=\sum_{i=1}^{N} \lambda_{i} \quad z\left(x_{i}\right), \operatorname{com} \sum_{i=1}^{N} \lambda_{i}=1
$$

em que $Z *\left(x_{0}\right)$ é o valor estimado para o local $\left(x_{0}\right)$ não amostrado; $z\left(x_{i}\right)$ é o valor obtido por amostragem no campo; $N$ é o número de amostras vizinhas e $\lambda i$ são os pesos aplicados em cada $z\left(x_{i}\right)$.

\section{RESULTADOS E DISCUSSÃO}

Os atributos carbono e argila foram estudados nas profundidades de 0-0,20 m e 0,40-0,60 m, representando os horizontes $\mathrm{A}$ e $\mathrm{B}$, respectivamente. Os parâmetros da estatística descritiva desses atributos são apresentados no Quadro 1 .
Na primeira profundidade (0-0,20 m), não houve diferenças significativas $(\mathrm{P}<0,05)$ entre as microbacias para o teor de carbono, enquanto, os teores de argila foram iguais entre as microbacias 1 e 2 e entre as microbacias 2 e 3 , porém a média da microbacia 4 diferiu significativamente de todas as das outras microbacias. (Quadro 1).

Os teores médios de carbono observados nas microbacias foram semelhantes aos resultados encontrados por Botschek et al. (1996) e Ruivo et al. (2002) em Latossolos Amarelos na Amazônia, entretanto, foram bem inferiores às médias encontradas por Laurance et al. (1999) e McGrath et al. (2001). Isso ocorreu, provavelmente, devido à escala de abordagem e a características físicas como textura e tipo de solo pesquisado.

Quadro 1 - Estatística descritiva dos atributos do solo na profundidade de 0 a 0,20 e 0,40 a 0,60 m nas microbacias estudadas Table 1 -Descriptive statistics of soil attributes at depths from 0 to 0.20 and 0.40 to $0.60 \mathrm{~m}$ in watersheds

\begin{tabular}{|c|c|c|c|c|c|c|c|c|c|}
\hline \multicolumn{10}{|c|}{ Parâmetros estatísticos dos atributos do solo $(0$ a $0,20 \mathrm{~m})$} \\
\hline Variável & Micr. & $\mathrm{N}$ & Méd. & $\mathrm{DP}$ & $\mathrm{CV}$ & Mín. & Máx. & Var. & $\bar{n}$ \\
\hline & 1 & 43 & $9,82^{\mathrm{a}}$ & 1,59 & 16,2 & 7,2 & 14,3 & moderada & 10 \\
\hline $\mathrm{C}$ & 2 & 65 & $10,00^{\mathrm{a}}$ & 2,44 & 24,4 & 5,9 & 18,1 & moderada & 23 \\
\hline \multirow[t]{3}{*}{$(\mathrm{g} / \mathrm{kg})$} & 3 & 39 & $9,94^{\mathrm{a}}$ & 1,74 & 17,5 & 6,8 & 14,9 & moderada & 12 \\
\hline & 4 & 38 & $10,49^{a}$ & 2,49 & 23,7 & 7,3 & 16,9 & moderada & 22 \\
\hline & Total & 185 & 10,05 & 2,14 & 21,3 & 5,9 & 18,1 & moderada & 17 \\
\hline \multirow{5}{*}{$\begin{array}{l}\text { Argila } \\
(\mathrm{g} / \mathrm{kg})\end{array}$} & 1 & 43 & $265,53^{\mathrm{a}}$ & 30,92 & 11,6 & 166,0 & 317,0 & baixa & 5 \\
\hline & 2 & 65 & $280,74^{\mathrm{ab}}$ & 33,00 & 11,8 & 228,0 & 407,0 & baixa & 5 \\
\hline & 3 & 39 & $297,50^{\mathrm{b}}$ & 60,35 & 20,3 & 166,0 & 444,0 & moderada & 16 \\
\hline & 4 & 38 & $422,39^{c}$ & 52,12 & 12,3 & 352,0 & 570,0 & baixa & 6 \\
\hline & Total & 185 & 309,84 & 72,81 & 23,5 & 166,0 & 570,0 & moderada & 21 \\
\hline \multicolumn{10}{|c|}{ Parâmetros estatísticos dos atributos do solo $(0,40$ a $0,60 \mathrm{~m})$} \\
\hline Variável & Micr. & $\mathrm{N}$ & Méd. & DP & $\mathrm{CV}$ & Mín. & Máx. & Var. & $n$ \\
\hline \multirow{5}{*}{$\begin{array}{l}\mathrm{C} \\
(\mathrm{g} / \mathrm{kg})\end{array}$} & 1 & 43 & $5,00^{\mathrm{a}}$ & 1,04 & 20,8 & 1,6 & 8,7 & moderada & 17 \\
\hline & 2 & 65 & $5,30^{\mathrm{ab}}$ & 1,37 & 25,8 & 3,1 & 14,2 & moderada & 26 \\
\hline & 3 & 39 & $5,28^{\mathrm{ab}}$ & 0,81 & 15,3 & 3,3 & 8,0 & moderada & 9 \\
\hline & 4 & 38 & $5,78^{b}$ & 1,29 & 22,3 & 4,0 & 10,0 & moderada & 19 \\
\hline & Total & 185 & 5,33 & 1,20 & 22,5 & 1,6 & 14,2 & moderada & 19 \\
\hline \multirow{5}{*}{$\begin{array}{l}\text { Argila } \\
(\mathrm{g} / \mathrm{kg})\end{array}$} & 1 & 43 & $382,63^{a}$ & 74,00 & 19,3 & 261,0 & 503,0 & moderada & 14 \\
\hline & 2 & 65 & $446,64^{b}$ & 32,80 & 7,3 & 381,0 & 546,0 & baixa & 2 \\
\hline & 3 & 39 & $439,72^{b}$ & 41,26 & 9,4 & 305,0 & 519,0 & baixa & 3 \\
\hline & 4 & 38 & $436,18^{\mathrm{b}}$ & 33,50 & 7,7 & 380,0 & 503,0 & baixa & 2 \\
\hline & Total & 185 & 428,15 & 53,37 & 12,5 & 261,0 & 546,0 & baixa & 6 \\
\hline
\end{tabular}

Micr. - microbacia; N - número de observações; Méd. - média; DP - desvio-padrão; CV - coeficiente de variação (\%); Mín. - valor mínimo do atributo; Máx. - valor máximo do atributo; Var. - variabilidade dos atributos de solo; segundo Wilding e Drees (1983), é dada por: CV < 15\% (baixa), CV 15-35\% (moderada) e CV > 35\% (alta); $n$ - número necessário de amostras para a obtenção de dados confiáveis na estatística clássica.

Médias seguidas de mesma letra não diferem significativamente entre si, pelo teste de Tukey $(\alpha=0,05)$.

R. Árvore, Viçosa-MG, v.31, n.1, p.83-92, 2007 
Alguns estudos têm descrito a relação significativa da argila com outros atributos do solo. Laurance et al. (1999), por exemplo, relataram que, na profundidade de $0-0,20 \mathrm{~m}$, o conteúdo de argila está positivamente correlacionado com a matéria orgânica e a biomassa, o que também foi observado por McGrath et al. (2001).

No subsolo $(0,40-0,60 \mathrm{~m})$, a microbacia 2 repetiu a alta dispersão do teor de carbono apresentada na profundidade de $0-0,20 \mathrm{~m}$, com maior valor de CV. Os teores de carbono dos horizontes A e B das microbacias apresentaram classe moderada de CV (Quadro 1), quando seria esperada classe alta, segundo estudos de Wilding e Drees (1983), porém o teor de argila da profundidade de $0-0,20$ m demonstrou classe moderada de CV, conforme previsto por esses autores.

A avaliação do CV em estudos de solo assume grande importância, pois, à medida que a variabilidade de um atributo aumenta, torna-se necessário um grande número de amostras para a obtenção de dados confiáveis (WARRICK e NIELSEN, 1980; DAVIS et al., 1995).
Neste estudo, ficaram evidenciadas diferenças texturais entre o horizonte superficial e o subsuperficial nas microbacias, com acúmulo de argila em profundidade; fato esse destacado por Sombroeck (1984), em pesquisas com solos amazônicos. Especificamente, no caso das microbacias 1, 2 e 3, houve maior diferença textural entre os horizontes A e B do que na microbacia 4. Nesta, ocorreu pouco acúmulo de argila no horizonte subsuperficial em relação à superfície, caracterizando maior homogeneidade textural.

\subsection{Análise da Distribuição Espacial dos Atributos Teor de Carbono e Argila}

Os atributos estudados foram submetidos à aplicação de semivariograma, com a finalidade de avaliar sua dependência espacial. Assim, as variáveis foram analisadas e ajustadas a modelos de semivariograma, considerandose individualmente cada microbacia e também o conjunto, representado por 185 amostras de cada profundidade. Os parâmetros dos semivariogramas dos teores de carbono do solo e da argila, em duas profundidades, são apresentados no Quadro 2.

Quadro 2 - Parâmetros dos semivariogramas das microbacias na profundidade de 0 a 0,20 e 0,40 a 0,60 m Table 2 -Semivariogram parameters of the watersheds at depths from 0 to 0.20 and 0.40 to $0.60 \mathrm{~m}$

\begin{tabular}{|c|c|c|c|c|c|c|c|}
\hline \multirow[t]{2}{*}{ Variável } & \multirow[t]{2}{*}{ Micr. } & Modelo & \multicolumn{3}{|c|}{ Efeito Pepita $\left(\mathrm{C}_{0}\right)$ Patamar $\left(\mathrm{C}+\mathrm{C}_{0}\right)$ Alcance $(\mathrm{m})$} & \multirow[t]{2}{*}{ Relação } & \multirow[t]{2}{*}{$\mathrm{DE}$} \\
\hline & & \multicolumn{4}{|c|}{ Profundidade $(0$ a $0,20 \mathrm{~m})$} & & \\
\hline & 1 & exponencial & 0,62 & 2,01 & 194,70 & 30,85 & Mo \\
\hline $\mathrm{C}$ & 2 & esférico & 1,76 & 6,34 & 138,60 & 27,76 & Mo \\
\hline \multirow[t]{4}{*}{$(\mathrm{g} / \mathrm{kg})$} & 3 & exponencial & 0,85 & 3,28 & 98,52 & 25,91 & Mo \\
\hline & 4 & esférico & 3,84 & 11,74 & 120,56 & 32,71 & Mo \\
\hline & Todas & exponencial & $\mathbf{0 , 4 5 0}$ & 3,8370 & 63,60 & 11,73 & Fo \\
\hline & 1 & exponencial & 388,00 & 974,10 & 144,21 & 39,83 & Mo \\
\hline Argila & 2 & exponencial & 88,00 & 1575,00 & 188,18 & 5,59 & Fo \\
\hline \multirow[t]{5}{*}{$(\mathrm{g} / \mathrm{kg})$} & 3 & esférico & 4,40 & 69,80 & 122,77 & 6,30 & Fo \\
\hline & 4 & esférico & 290,00 & 5690,00 & 120,56 & 5,10 & Fo \\
\hline & Todas & esférico & 520,00 & $10.150,00$ & 398,00 & 5,12 & Fo \\
\hline & & \multicolumn{6}{|c|}{ Profundidade $(0,40$ a $0,60 \mathrm{~m})$} \\
\hline & 1 & esférico & 0,364 & 0,799 & 194,70 & 45,56 & Mo \\
\hline $\mathrm{C}$ & 2 & esférico & 0,001 & 0,616 & 37,60 & 0,16 & Fo \\
\hline \multirow[t]{4}{*}{$(\mathrm{g} / \mathrm{kg})$} & 3 & esférico & 0,002 & 0,175 & 20,20 & 1,14 & Fo \\
\hline & 4 & esférico & 0,245 & 1,991 & 120,56 & 12,31 & Fo \\
\hline & Todas & exponencial & 0,7440 & 1,7330 & 398,00 & 42,93 & Mo \\
\hline & 1 & esférico & 1150,00 & 9206,00 & 193,60 & 12,49 & Fo \\
\hline Argila & 2 & esférico & 604,00 & 1667,00 & 188,18 & 36,23 & Mo \\
\hline \multirow[t]{3}{*}{$(\mathrm{g} / \mathrm{kg})$} & 3 & exponencial & 1,00 & 855,10 & 56,10 & 0,12 & Fo \\
\hline & 4 & esférico & 1,00 & 1133,00 & 26,40 & 0,09 & Fo \\
\hline & Todas & esférico & 955,00 & $2.048,00$ & 237,10 & 46,63 & Mo \\
\hline
\end{tabular}

A classificação da dependência espacial (DE) das propriedades de solo, segundo Cambardella (1994), é estimada pela relação $\left[\mathrm{C}_{0} /\left(\mathrm{C}+\mathrm{C}_{0}\right)\right] \times 100$, como: $\leq 25 \%$ - Forte (Fo); entre 25 e $75 \%$ - Moderada (Mo) e $>75 \%$ - Fraco (Fr). 
Os atributos estudados apresentaram dependência espacial na profundidade de 0-0,20 m, dentro de um alcance variando de $63,60 \mathrm{~m}$ para o carbono até um máximo de 398 m para a fração argila (Quadro 2). Conforme sugestão de Cambardella et al. (1994), as propriedades carbono do solo e argila foram classificadas de acordo com a magnitude da sua dependência espacial, levandose em consideração o efeito pepita observado. Por esse critério, quanto menor a proporção do efeito pepita em relação ao patamar do semivariograma, maior a continuidade do fenômeno e maior a dependência espacial apresentada pela variável.

Paisagens aparentemente homogêneas podem causar equívocos na avaliação ou manejo, se não forem corretamente analisadas. Cerri et al. (2004a), utilizando funções geoestatísticas, observaram uma heterogeneidade muito grande entre os atributos de solo no Estado de Rondônia, apesar da aparente semelhança entre os locais estudados. Nesse estudo, os autores utilizaram amostragens com distância de $25 \mathrm{~m}$ em malha regular, observando grande efeito pepita na maioria dos atributos. Essa é uma típica situação em que alta variabilidade existe dentro de um pequeno espaço, inclusive dentro da distância inferior à das coletas.

Na profundidade de $0,40-0,60 \mathrm{~m}$, relativa ao horizonte diagnóstico, as microbacias 1 e 2, apesar de apresentarem uma composição de solos bem distinta entre si, mostraram um alcance para o teor de argila bem superior ao das outras microbacias. Aparentemente, o maior alcance do teor de argila para a microbacia 1 não se justifica, pois essa bacia apresenta o mais variado universo pedológico. Pode-se aventar que os processos de gênese do solo atuantes nesse contexto (latolização e podzolização, associado à plintização) possibilitaram a micro-diversidade de ambientes muito bem definidos, isto é, grande variação do conteúdo de argila (261 até 503 g.kg-1) confinada a pequenos espaços (Quadro 2).

De modo geral, os teores de carbono tendem a ser mais altos nas partes baixas da paisagem, exceto na microbacia 1 . Isso, provavelmente pelo fato dessa microbacia ser muito heterogênea quanto às classes de solo e em razão dos aspectos relativos a seu formato, com diminuta área de deposição. Essas diferenças podem ser expressas pelo fator forma (F), que, segundo Resende et al. (2002), é obtido pela relação: $\mathrm{F}=\mathrm{A} / \mathrm{L}^{2}$, em que A é a área $\left(\mathrm{m}^{2}\right)$ e L é o comprimento da microbacia (m).
Esse fator gera informações acerca das características das bacias, como a forma mais alongada ou mais circular, o que contribui para delinear a dinâmica no seu interior. Os fatores forma das microbacias são: $\mathrm{F}$ (microbacia $1)=1,2 ; \mathrm{F}($ microbacia 2$)=0,4 ; \mathrm{F}($ microbacia 3$)=0,8$; e $\mathrm{F}($ microbacia 4$)=0,7$.

\subsection{Estoques de Carbono do Solo das Microbacias}

Os solos das microbacias apresentaram aumento da densidade aparente do horizonte A para o horizonte $\mathrm{B}$, principalmente na ocorrência do horizonte B textural (Bt). A média da densidade aparente nos horizontes de superfície foi de $1,36 \pm 0,081 \mathrm{~g} . \mathrm{cm}^{-3}$, enquanto nos horizontes subsuperficiais a média foi de 1,46 $\pm 0,083$ g.cm ${ }^{-3}$ (Figura 1).

Esses valores são semelhantes aos obtidos por Moraes et al. (1996), que encontraram a variação de $1,30 \mathrm{~g} . \mathrm{cm}^{-3}$ nos primeiros $0,05 \mathrm{~m}$ do solo e $1,51 \mathrm{~g} . \mathrm{cm}^{-}$ ${ }^{3}$ na profundidade de $0,20-0,30 \mathrm{~m}$. Também Melo (2003), estudando solos do estado do Acre, encontrou 1,10 g. $\mathrm{cm}^{-3}$ nos horizontes superficiais e $1,50 \mathrm{~g} . \mathrm{cm}^{-3}$ no subsolo.

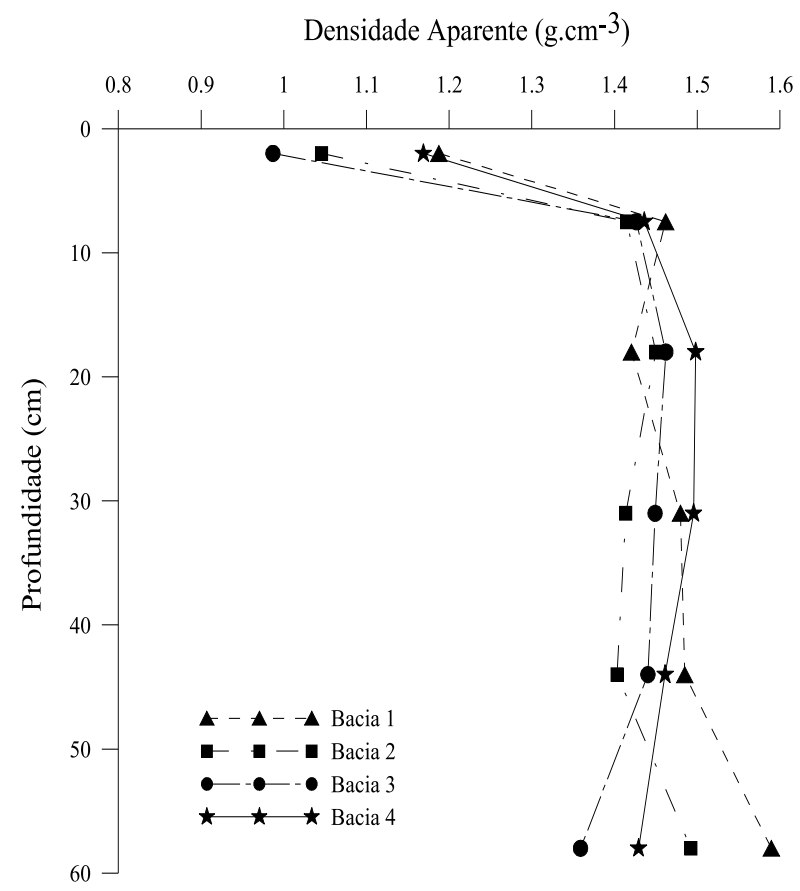

Figura 1 - Densidade aparente média das microbacias com a profundidade.

Figure 1 -Average apparent density of watersheds with the depth. 
As médias dos estoques de carbono nas profundidades de 0-0,20 m e 0-0,30 m (Quadro 3) indicam que, na primeira profundidade $(0-0,20 \mathrm{~m})$, houve muita semelhança com os resultados obtidos por Cerri et al. (2004b) utilizando modelagem geoestatística, em Rondônia. Da mesma forma, na camada de 0-0,30 m o estoque de carbono encontrado nas microbacias foi compatível com os estudos apresentados por Moraes et al. (1996) e Melo (2003).

Neill et al. (1997) encontraram estoques de carbono semelhantes ao deste estudo em algumas localidades de Rondônia e também valores superiores em outras regiões. Porém, Batjes e Dijkshoorn (1999) e Bernoux et al. (2002) observaram, na profundidade de 0-0,30 $\mathrm{m}$, valores superiores de estoque de carbono em solos amazônicos sob floresta do que os encontrados neste trabalho.

O maior estoque de carbono nas primeiras camadas do solo é um comportamento esperado, já que os

Quadro 3-Média do estoque de carbono $\left(\right.$ t.ha $\left.^{-1}\right)$ nas microbacias em profundidades de 0 a $0,20 \mathrm{~m}$ e 0 a $0,30 \mathrm{~m}$

Table 3 -Average of carbon stock $\left(t h \mathrm{~h}^{-1}\right)$ in watersheds at depths 0 to $0.20 \mathrm{~m}$ and 0 to $0.30 \mathrm{~m}$

\begin{tabular}{lccc}
\hline Variável & Microbacia & N. $^{\text {o de observações }}$ & Média \\
\hline \multirow{3}{*}{ C $\left(\right.$ t.ha $\left.^{-1}\right)$} & 1 & 43 & 27,10 \\
0 a $0,20 \mathrm{~m}$ & 2 & 65 & 26,20 \\
& 3 & 39 & 26,80 \\
& 4 & 38 & 29,60 \\
\cline { 2 - 4 } & Total & $\mathbf{1 8 5}$ & $\mathbf{2 7 , 2 0}$ \\
\hline \multirow{3}{*}{ C $\left(\right.$ t.ha $\left.^{-1}\right)$} & 1 & 43 & 34,30 \\
0 a $0,30 \mathrm{~m}$ & 3 & 65 & 35,30 \\
& 4 & 39 & 35,40 \\
& & 38 & 38,50 \\
\cline { 2 - 4 } & Total & $\mathbf{1 8 5}$ & $\mathbf{3 5 , 7 0}$ \\
\hline
\end{tabular}

horizontes superiores sofrem maior influência da matéria orgânica depositada pela floresta, principalmente pela morte de árvores grandes. É também nessa região do perfil que a ciclagem de nutrientes é predominante, onde os principais processos de troca são mais intensos, alimentados pela deposição da serrapilheira na superfície do solo (SCHUMACHER et al., 2003; VITAL et al., 2004). Porém, apesar de o teor de carbono decrescer com a profundidade, o estoque não seguiu essa tendência com a mesma intensidade, pois o aumento da densidade aparente com a profundidade compensou aquela diminuição no teor.

Os modelos ajustados de semivariograma consideraram a quantidade de 185 pontos representando as quatro microbacias, o que melhorou a confiabilidade da modelagem geoestatística, comparando-se com modelos obtidos para cada microbacia, isoladamente. Essa constatação confirmou a melhoria da aplicação da geoestatística quando foi empregada quantidade maior de pontos observados (WEBSTER e OLIVER, 1992).

O modelo de semivariograma que melhor se ajustou ao estoque de carbono (t.ha $\left.{ }^{-1}\right)$ na profundidade de 0 $0,60 \mathrm{~m}$ foi o exponencial, com alcance $\left(\mathrm{A}_{0}\right)$ de 185,40 $\mathrm{m}$ e moderada dependência espacial. Dessa forma, $\mathrm{o}$ alcance foi muito superior ao espaçamento de amostragem adotado $(20 \times 20 \mathrm{~m})$, o que rejeitou a hipótese de aleatoriedade dos dados.

No Quadro 4 são apresentados os parâmetros estatísticos do estoque de carbono nas quatro microbacias, considerando-se um perfil de 0-0,60 m. A média do estoque de carbono foi de 59,74 $\pm 10,30$ t.ha ${ }^{-1}$. A Figura 2 ilustra a distribuição espacial na paisagem das microbacias.

Quadro 4 - Estatística descritiva do estoque de carbono das microbacias na profundidade de 0 a $0,60 \mathrm{~m}$ Table 4-Descriptive statistics of carbon stock of watersheds at depth 0 to $0.60 \mathrm{~m}$

\begin{tabular}{lccccccc}
\hline & \multicolumn{7}{c}{ Parâmetros estatísticos do estoque de carbono $(0$ a $0,60 \mathrm{~m})$} \\
\hline Variável & Microbacia & $\mathrm{N}$ & Média & DP & CV & Mín. & Máx. \\
\hline \multirow{3}{*}{} & 1 & 43 & $56,73^{\mathrm{a}}$ & 8,30 & 14,6 & 40,65 & 84,57 \\
$\left(\right.$ t.ha $\left.^{-1}\right)$ & 2 & 65 & $59,35^{\text {ab }}$ & 11,03 & 18,6 & 38,71 & 109,17 \\
& 3 & 39 & $59,22^{\text {ab }}$ & 9,59 & 16,2 & 43,62 & 93,46 \\
& 4 & 38 & $64,35^{\text {b }}$ & 10,57 & 16,4 & 48,43 & 90,31 \\
\cline { 2 - 8 } & Total & $\mathbf{1 8 5}$ & $\mathbf{5 9 , 7 4}$ & $\mathbf{1 0 , 3 0}$ & 17,2 & $\mathbf{3 8 , 7 1}$ & $\mathbf{1 0 9 , 1 7}$ \\
\hline
\end{tabular}

$\mathrm{N}$ - número de observações; média seguida de mesma letra não difere significativamente entre si, pelo teste de Tukey $(\mathrm{a}=0,05)$; DP desvio-padrão; e CV - coeficiente de variação (\%). 


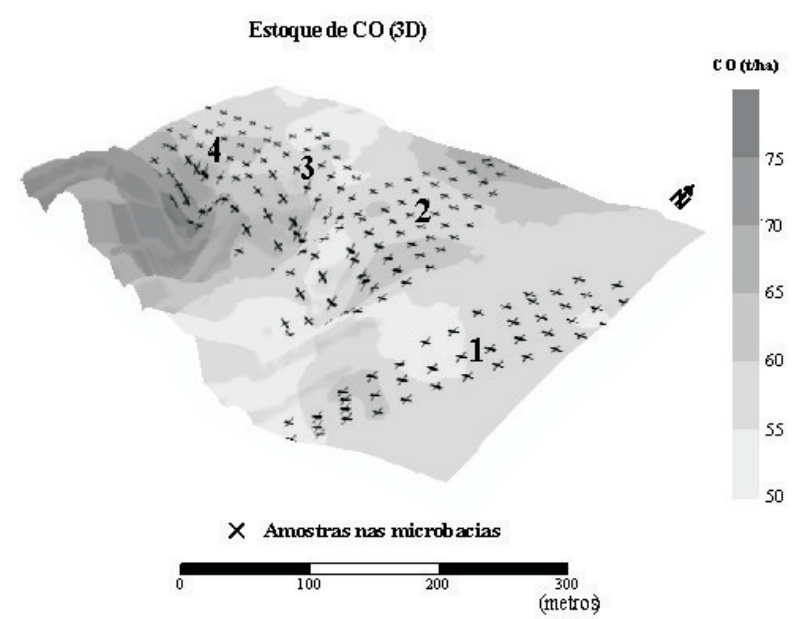

Figura 2 - Distribuição espacial do estoque de carbono nas microbacias na profundidade de (0 a $0,60 \mathrm{~m})$.

Figure 2-Spatial distribution of carbon stock in watersheds at depth 0 to $0.60 \mathrm{~m}$.

Analisando individualmente as microbacias, observou-se que a microbacia 1 possuía o menor estoque médio de carbono no solo e a microbacia 4, o maior, apesar de não haver diferenças significativas entre as microbacias 1,2 e 3. É importante destacar que a microbacia 1 também possuía os menores teores de argila nas duas profundidades, evidenciando-se a influência que a fração argila exerce no estoque de carbono (LAURANCE et al., 1999; McGRATH et al., 2001).

\section{CONCLUSÕES}

a) $\mathrm{O}$ atributo carbono apresentou variabilidade moderada nas camadas A e B do solo, em todas as microbacias. Isso demonstra que a extrapolação de dados para estimativas de estoque de carbono em áreas maiores pode projetar resultados equivocados quando a variabilidade espacial de atributos de solo não é levada em consideração.

b) Todos os atributos estudados apresentaram dependência espacial dentro da escala de espaço observada (20 x $20 \mathrm{~m})$, tanto no horizonte superficial quanto no subsuperficial, ou seja, nesse espaço de coleta de amostras os atributos não são independentes.

c) A modelagem aplicada permitiu estimar o estoque de carbono das quatro microbacias, bem como o alcance e a magnitude da dependência espacial de cada atributo estudado. Além disso, foi possível identificar áreas homogêneas na escala de microbacias, demonstradas por meio de mapas de distribuição espacial.

\section{AGRADECIMENTOS}

Ao Comitê de Treinamento do Programa LBA (Experimento de Grande Escala Biosfera e Atmosfera na Amazônia), pelo incentivo e pela oportunidade, bem como ao CNPq, pela concessão de bolsas (proc. institucional CNPq 680.051/03-1).

\section{REFERÊNCIAS}

BATJES, N. H.; DIJKSHOORN, J. A. Carbon and nitrogen stocks in the soils of the Amazon Region. Geoderma, v.89, p.273-286, 1999.

BERNOUX, M. et al. Brazil's soil carbon stocks. Soil Science Society of America Journal, v.66, p.888-896, 2002.

BOTSCHEK, J. et al. Soil chemical properties of a toposequence under primary rain forest in the Itacoatiara vicinity (Amazonas, Brazil).

Geoderma, v.72, p.119-132, 1996.

BRASIL. Ministério das Minas e Energia. Secretaria Geral. Projeto RADAMBRASIL. Folha SC. 21 - Juruena. Rio de Janeiro: MMESG, 1980. 460p. (Levantamento de Recursos Naturais, 20).

CAMARgo, O. A. et al. Métodos de análise química, mineralógica e física de solos do Instituto Agronômico de Campinas. Campinas: Instituto Agronômico, 1986.94p. (Boletim Técnico, 106)

CAMBARDELLA, C. A. et al. Field-scale variability of soil properties in Central Iowa soils. Soil Science Society of America Journal, v.58, p.1501-1511, 1994.

CERRI, C. E. P. et al. Assessment of soil property spatial variation in an Amazon pasture: basis for selecting an agronomic experimental area.

Geoderma, v.123, p.51-68, 2004a.

CERRI, C. E. P.; CERRI, C. C.; PAUSTIAN, K.; BERNOUX, M.; MELLILO, J. M. Combining soil C and $\mathrm{N}$ spatial variability and modeling approaches for measuring and monitoring soil carbon sequestration. Environmental

Management, v.33, S.1, p.274-288, 2004b. 
COUTO, E. G.; OLIVEIRA, V. A. Levantamento de reconhecimento de alta intensidade dos solos e avaliação da aptidão agrícola das terras do imóvel da Rohden Industria Lignea LTDA, município de Juruena/MT. Cuiabá: UFMT/IBGE, 2003. 92p.

DAVIS, J. G. et al. Variability of soil chemical properties in two sandy, dunal soils of Niger. Soil Science, v.159, p.321-330, 1995.

EMPRESA BRASILEIRA DE PESQUISA AGROPECUÁRIA - EMBRAPA. Centro Nacional de Pesquisa de Solos. Manual de métodos de análise de solo. 2 . ed. revista e atualizada. Rio de Janeiro: 1997. 212p.

EMPRESA BRASILEIRA DE PESQUISA AGROPECUÁRIA - EMBRAPA. Centro Nacional de Pesquisa de Solos. Sistema brasileiro de classificação de solos. Rio de Janeiro: 1999. 412p.

INSTITUTO BRASILEIRO DE GEOGRAFIA E ESTATÍSTICAS - IBGE. Projeto zoneamento das potencialidades dos recursos naturais da Amazônia Legal. Departamento de Recursos Naturais e Estudos Ambientais. Convênio IBGE/SUDAM. Rio de Janeiro: 1990. 212p.

LAURANCE, W. F. et al. Relationship between soils and Amazon forest biomass: a landscapescale study. Forest Ecology and Management, v.118, p.127-138, 1999.

McGRATH, D. A. et al. Effects of land-use change on soil nutrient dynamics in Amazônia.

Ecosystems, v.4, p.625-645, 2001.

MELO, A. W. F. Avaliação do estoque e composição isotópica do carbono do solo no Acre. 2003. 74 f. Dissertação (Mestrado em Ecologia de Agroecossistemas) Escola Superior de Agricultura Luiz de Queiroz, Piracicaba, 2003.

MORAES, J. F. L. et al. Soil properties under Amazon forest and changes due to pasture installation in Rondônia, Brazil. Geoderma, v.70, p.63-81, 1996.
NEILL, C. et al. Soil carbon and nitrogen stocks following forest clearing for pasture in the southwestern Brazilian Amazon. Ecological Applications, v.7, n.4, p.1216-1225, 1997.

RESENDE, M. et al. Pedologia: base para distinção de ambientes. 3. ed. Viçosa, MG: NEPUT, 2002. 338p.

ROBERTSON, G. P. GS+: Geostatistic for the enviroment sciences - GS+ user's guide version 5 . Plainwell, Gamma Desing Software, 2000. 200p.

RUIVO, M. L. P. et al. The soils of the LBA experimental sites (Caxiuanã, Pará State, Brazil). In: WCSS, 17. Anais eletrônicos... Thailand: 2002. Disponível em: <http:// www.ldd.go.th/Wcss2002/papers/2152.pdf>. Acesso em: 20 out. 2003.

SCHUMACHER, M. V. et al. Retorno de nutrientes via deposição de serapilheira em um povoamento de acácia-negra (Acacia mearnsii De Wild.) no Estado do Rio Grande do Sul. Revista Árvore, v.27, n.6, p.791-798, 2003.

SOMBROECK, W. G. Soils of the Amazon Region. In: SIOLI, H. 1984. The Amazon, limnology and landscape ecology of a mighty tropical river and its basin. Dordrecht/Boston/Lancaster: Dr. W. Junk Publishers, 1984. p.521-536.

STATISTICAL PACKAGE FOR THE SOCIAL SCIENCES - SPSS. SPSS for Windows. Base User's Guide, Release 10. Chicago: 1999.

STEIN, A.; ETTEMA, C. An overview of spatial sampling procedures and experimental design of spatial studies for ecosystem comparisons.

Agriculture, Ecosystems and Environment, v.94, p.31-47, 2003.

SURFER. Surfer 8.0: Contouring and 3d surface mapping for scientists and engineers. User's Guide. New York: Golden Software, 2002. 619p.

TRANGMAR, B. B.; YOST, R. S.; UEHARA, G. Application of geostatistics to spatial studies of soil properties. Advances in Agronomy, v.38, p.45-94, 1985.

R. Árvore, Viçosa-MG, v.31, n.1, p.83-92, 2007 
VITAL, A. R. T. et al. Produção de serapilheira e ciclagem de nutrientes de uma floresta estacional semidecidual em zona ripária. Revista Árvore, v.28, n.6, p.793-800, 2004.

WARRICK, A. W.; NIELSEN, D. R. Sapatial variability of soil physical properties in the field. In: HILLEL, D. (Ed.). Application of soil physics. New York: Academic Press, 1980. p.319-344.
WEBSTER, R.; OLIVER, M. A. Sample adequately to estimate variograms of soil properties. Journal of Soil Science, v.43, p.177-192, 1992.

WILDING, L. P.; DREES, L. R. Spatial variability and pedology. In: Wilding, L.P. et al. (Ed.).

Pedogenesis and Soil Taxonomy I. Concepts and Interactions. Amsterdam: Elsevier, 1983. p.83-116. 